\title{
Implementation of Wide Area Monitoring Systems and Laboratory-Based Deployment of PMUs
}

\author{
Mohammad Golshani \\ Gareth A. Taylor \\ Ioana Pisica \\ Phillip Ashton \\ mohammad.golshani@brunel.ac.uk gareth.taylor@brunel.ac.uk ioana.pisica@brunel.ac.uk phillip.ashton@brunel.ac.uk
}

Brunel Institute of Power Systems, Brunel University, London, UK

\begin{abstract}
Power systems are operating in a more complicated condition and therefore encounter more challenges. If one part of a power grid becomes seriously out of synchronism with the rest, the whole network can become unstable and blackout may occur. In this regard, we need to use advanced and smart monitoring tools to quickly and reliably observe the changing state of the key electrical parameters in real time, take appropriate corrective measures and isolate faults. Phasor Measurement Units (PMUs) by employing satellite technology, offer a state-of-the-art Wide Area Monitoring System (WAMS) for improving power system monitoring, control and protection. In this paper, we present information about WAMS components and requirements as well as description of work carried out in Brunel University laboratory to develop and implement a synchronized measurement system.
\end{abstract}

Index Terms-Open source, Phasor Data Concentrator (PDC), Phasor Measurement Unit (PMU), Synchrophasor, WAMS.

\section{INTRODUCTION}

Reliability is one of the prominent factors in operation of power systems that has notable economic impact and influence on society. Historically, power systems have been remarkably reliable. Although minor outages have been common, large-scale and wide-spread outages rarely happened and interruptions have occurred over relatively limited area. However, changes in the wholesale electricity market alongside the difficulties in upgrading the transmission systems have caused power systems to face more challenging network wide issues. In this condition, a minor disturbance can be intensified by a series of events leading to network wide effect. Subsequently, system may completely collapse, if timely actions are not adopted [1].

The current Supervisory Control and Data Acquisition (SCADA) systems observe grid conditions every few seconds. Thus they are incapable of providing information about the dynamic state of the power system. In addition, SCADA data are not consistently time-synchronized and shared widely across the network. Therefore, SCADA does not provide operators with real-time and wide-area visibility. The emergence of PMUs provides a significant improvement in reliability by offering unprecedented time-synchronized and high resolution information over a wide-area, in realtime. Many advanced smart grid applications can take advantage of the measurement capabilities of PMUs. These applications enable utilities to react promptly to the contingencies and prevent large-scale blackouts [2].
A PMU-based WAMS is a system in which PMUs measure power system parameters including frequency, voltage, and current phasors with high accuracy. Meanwhile, each phasor is time-stamped using signals from Global Positioning System (GPS) so that the microsecond when the measurement taken is permanently attached to it. Afterwards, the timecritical phasor data are collected from various locations in the electrical grid and will be transmitted to a central location called Phasor Data Concentrator (PDC). A PDC receives and time-synchronizes phasor data from geographically distributed PMUs and produces a real-time, time-aligned output data stream. This information will be exploited by smart grid applications [3]. These applications are generally categorized as on-line or off-line. On-line applications process real-time data as it arrives to the client system. In contrast, off-line applications process data that is archived [4]. Figure 1 illustrates the structure of WAMS and its components [5].

As described, WAMS comprises of four main parts: PMUs, communication networks, PDCs, and application software. A successful implementation of WAMS needs an elaborate planning and designation of equipment and methods to fulfil these four parts requirements. The rest of the paper is structured as follows: Section II describes the PMUs working principles and their installation requirements. Communication networks and related technologies are discussed in Section III. Section IV provides details about PDCs and their technical challenges. Common applications that benefit from synchrophasor measurements are addressed in Section V. Section VI presents information about Brunel University laboratory-based WAMS and examples of events captured. Finally, the paper is concluded in Section VII.

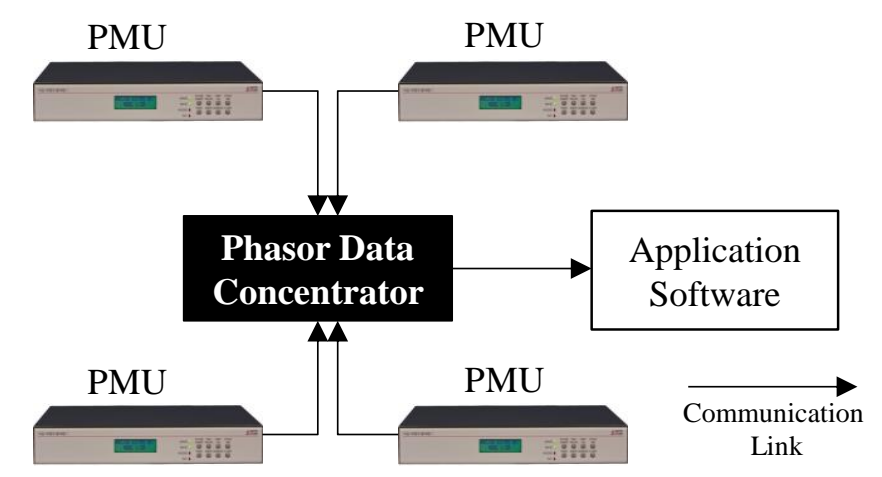

Fig. 1. WAMS structure 


\section{Phasor MEASUREMENT UNITS (PMUS)}

A phasor is a mathematical representation of an electrical waveform based on the amplitude and phase angle. Use of the phasor notation considerably simplifies not only the mathematics but also the electronics and processing requirement [6]. The most common technique for determining phasor representation of $\mathrm{AC}$ waveforms is to take data samples from the waveform using an analog to digital converter and apply the Discrete Fourier Transform (DFT) [7]. There is a wide variety of phasor measurement equipment available. Many PMUs are produced as dedicated devices, while there are also vendors that offer PMU functionality as a supplementary feature on their other products, such as relays and Digital Fault Recorders (DFRs) [8].

In most cases, PMU installation is for a permanent operation so all aspects should be considered in order to have a well-established measurement system. PMUs can be different in the matter of algorithm selection, timing input, number of voltage and current inputs, communication interface, accuracy, etc. [9]. However, they have number of common requirements, including: access to signals to be measured, a timing signal to synchronize the measurements, and a power supply [10].

PMUs need to have access to the voltage and current signals to be measured. In some substations these signals are available in a single location, while in many substations these signals are brought to the different buildings or cabinets. In this condition, it may be necessary to include several PMUs or use a PMU that has distributable input modules to cover the whole substation [10]. The analog input signals corresponding to voltages and currents are obtained from the instrument transformers as shown in Figure 2. Several instrument transformer technologies are available that can be used to transform signals to an appropriate level for PMU applications. It should be noted that the measurement accuracy of PMU is directly affected by the instrument transformers [11].

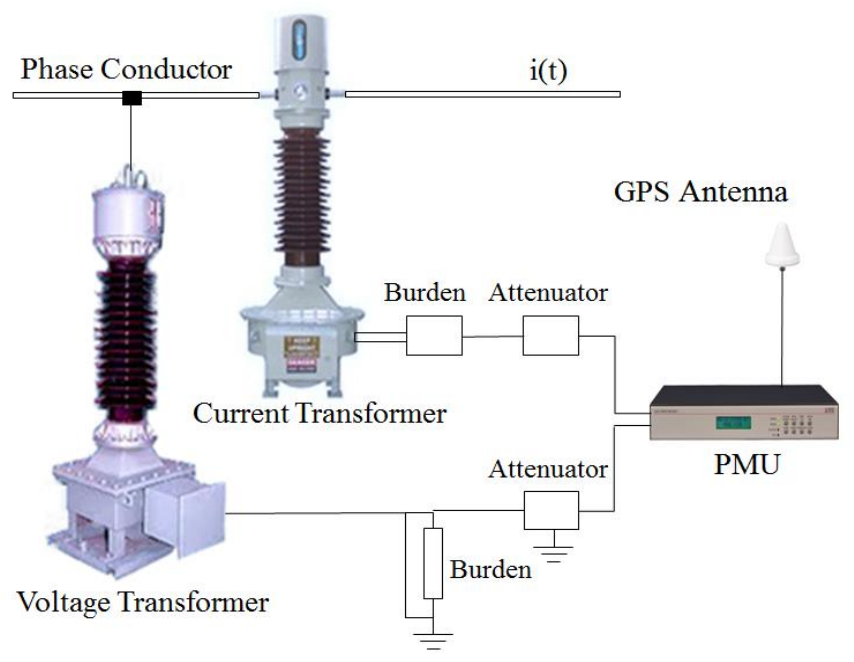

Fig. 2. Typical PMU installation and interconnection in substation [2]
Time synchronization of phasors is necessary in order to obtain a complete view of the grid at specific time and to make the comparison of measurements from PMUs installed at different geographical locations more accurate. Synchronized phasors are known as synchrophasors. In this regard, PMUs require a precise time input. This synchronization is achieved by using a sampling clock which is phase-locked to the one-pulse-per-second signal provided by a GPS receiver [7]. Most PMUs use a direct GPS input from an antenna or an IRIG-B time code. It is possible to use one GPS antenna for more than one PMU by using GPS antenna splitter. It should be noticed that if one of the PMUs provides antenna power, the splitter must have DC blocks on other PMUs side that do not supply power. Type and length of the cable are important as the signal attenuates rapidly in the cable. It is necessary to make sure that each PMU gets a strong enough signal from the antenna. The amplifier can be used to step up the low level signal to the acceptable level for PMUs [12].

The power supply for PMU comes from AC or DC source. But it should be noted that PMUs need to operate continuously, even and especially during power system disturbances. Thus, they must be connected to an uninterruptible power source [10].

To assure that the measurements from all PMUs are comparable under various power system operations and for the advancement of the connectivity and interoperability, standardization is an important requirement. Most PMUs on the market today use the IEEE C37.118 standard. The Synchrophasor and frequency values must meet the general definition as well as minimum accuracy requirements given in the standard [13].

\section{COMMUNICATION NETWORKS}

In developing a WAMS, reliable and high speed communication infrastructure that enables secure sharing of data among PMUs, PDCs and smart grid applications play an important role. IEEE C37.118 frames are typically not sent directly over network, but rather based on the concept of layered protocols they are encapsulated within frames of other communication protocols [14]. The speed of data transfer is less critical for the off-line applications, while for the on-line applications a faster data transfer is required and depends on the type of application. The communication infrastructure should therefore be able to support different Quality-of-Services (QoS) classes for traffic and should be able to prioritize one class over another [1].

Most PMUs have an Ethernet interface, although there are some models that only use asynchronous serial (RS 232). An interface device (modem, Router, etc.) is required between the PMU communication link and the communication system. Communication over network using Internet Protocol (IP) can be connection-oriented (TCP) or connectionless (UDP). TCP rearranges data packets in the order specified and retransmits lost or corrupted data. In the case of UDP there is no built-in 
ordering and recovery of data, but the transmission speed is higher than TCP. Therefore, UDP is used commonly with PMUs since a small amount of lost data is preferable over delayed data in many real-time smart grid applications [10].

Many utilities today use Multi-Protocol Label Switching (MPLS) Virtual Private Networks (VPNs), or Frame Relay technology for WANs. These communication media provide a guaranteed bit rate but are not guaranteed to be error free [14]. Since data may be shared with multiple entities at the same time and for communication redundancy, a WAMS should also support multicast data sharing. It is also crucial to secure WAMS in order to ensure the availability, integrity, and confidentiality of the data transmitted over network [1].

\section{PHASOR DATA CONCENTRATORS (PDCS)}

IEEE C37.118 standard defines four message types: data, configuration, header and command. The first three message types are transmitted from the data source, PMU or PDC, and the last one is received by the data source. It should be noted that PDC itself can be considered as a data source when it sends data to another PDC [15]. Data messages cannot stand alone, as they do not describe the data they contain. Configuration messages contain information required to determine the meaning of each individual field with the data message [14]. A typical exchange between a PMU and a PDC is as following [16]:

- The PDC sends a command message to the PMU to request human-readable description information

- The PMU replies with a header message

- The PDC sends a command message to the PMU to request configuration information

- The PMU replies with a configuration message

- The PDC sends a command message to the PMU to request data

- The PMU replies with data messages until the PDC sends a command message to terminate its request

A PDC collects data from multiple PMUs or other PDCs and time-aligns phasor data according to their time-stamps. In fact, it forms a data packet with a given time-stamp, and then assembles all data received with that time-stamp into the single packet. The produced real-time and synchronized output data stream can be exploited by smart grid applications and is also stored for future analysis. A high number of PMUs are installed in the whole power systems and they depend on the limited number of PDCs to process and store their data. Therefore, each PDC needs to deal with huge amount of data and the required data storage capacity should be considered. As more PMUs are deployed in the system, the number of measurement samples increases and the storage requirement accelerates [17]. Storing and processing a huge volume of PMUs measurements and scanning through terabytes of information to find the particular event will be a big challenge for WAMS. Currently available storage and processing systems, such as Storage Area Network (SAN) and Relational DataBase Management System (RDBMS), have low read rates and do not work well with high resolution time-series data of WAMS. Thus efficient platform is required to interact with huge amount of high-resolution data, such as Hadoop [18, 19].

\section{WAMS APPLICATIONS FOR POWER SYSTEMS}

WAMS brings great potential for improving reliability and stability based on the operational feature of the PMUs. In the literatures, there are a large number of WAMS applications; however they can be categorized into three main groups of monitoring, protection, and control applications [20, 21].

\section{A. Power System Monitoring}

A PMU-based monitoring systems offer various kinds of functions, such as wide area visualisation, model validation, state estimation, near real-time event replay, post event analysis, early warning of potential problems etc. These functionalities will significantly improve situational awareness. When power system operates in a normal condition, providing applications with reduced resolution synchrophasor measurements is sufficient. However, for the near real-time event replay mode and post event analysis, measurements with full resolution are required [22, 23].

\section{B. Power System Protection}

Synchronized phasor measurements offer solution to a number of complex protection problems and improve the performance of the protection applications. Phasor measurements are particularly effective in enhancing power system protection functions that have a slow response time requirement. Some protection systems that could benefit from PMUs include: adaptive dependability and security, back up protection of distance relays, adaptive out-of-step, angular voltage stability of network, etc [7].

\section{Power system control}

Prior to the emergence of real-time phasor measurements most of the control systems were processed locally due to low time delay. Controllers like variable series capacitor (VSC), universal power flow controllers (UPFCs) and power system stabilizers regulate the grid based on a local feedback. While phasor measurements in addition to remote control of the power systems, could provide direct feedback to these controllers and enable dynamic control of the power systems. Using PMUs to damp the low frequency inter-area oscillations is one of the effective applications of WAMS [7, 24].

\section{PMU-BASED MONITORING IN BRUNEL UNIVERSITY LABORATORY}

The high cost of early PMU devices, has historically restricted the use of PMU technology to the transmission systems. However, due to the recent developments, the cost of the components from which PMUs are assembled 
decreased dramatically. Hence deploying PMUs have become prevalent across the utility environment, including distribution systems [8]. Moreover, employing PMUs in laboratory-based and domestic level simplifies investigation of wide area monitoring system compared to the more complicated case of installing them in substations. There is more equipment required inside a substation for deploying PMUs, such as step-down transformers that bring the threephase voltage or current level to instrumentation level. Brunel University, London installed a PMU that is connected to the 3 phase $415 \mathrm{~V}$ AC domestic supply level and joined a laboratory-based WAMS. Currently, PMUs measured synchrophasors from 4 UK Universities are transmitted via the Internet to a server in Ljubljana, Slovenia. As the PMUs are well geographically distributed across the Scottish to England system, as depicted in Figure 3, they provide a relatively good visibility of events through the covered area.

\section{A. ELPROS PDC}

The PDC in Slovenia is running WAProtector, which is a system for wide area monitoring, protection and control provided by the ELPROS [25]. This application presents various details from the collected PMUs data, such as: phasor representation of voltages, phase angles geographic chart, two dimensional frequency, voltage magnitude and phase angle trending charts, angle difference and oscillation graphs and so on. The events details including the time, location and type of events are shown on the main display and also will be stored for future analysis.

Figures 4 and 5 show examples of identifying frequency and voltage deviations across UK network using PMUs collected data, which happened on 30 September 2012. Frequency is a good indicator of power system condition and the size of frequency deviation is well correlated with the severity of the event [2]. The voltage phase angle difference between nodes is another key factor in monitoring. Power

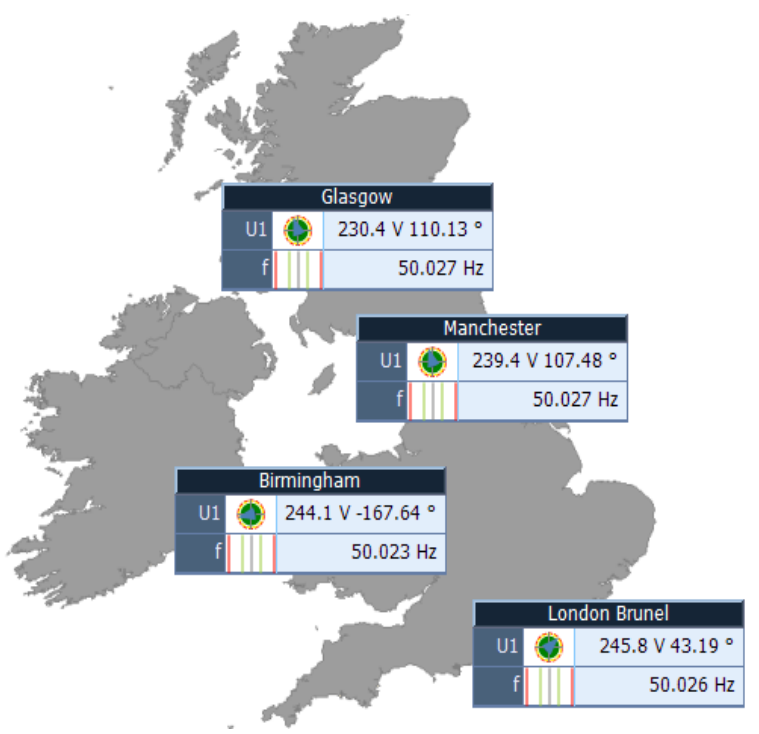

Fig. 3. The WAMS PMUs locations across the UK

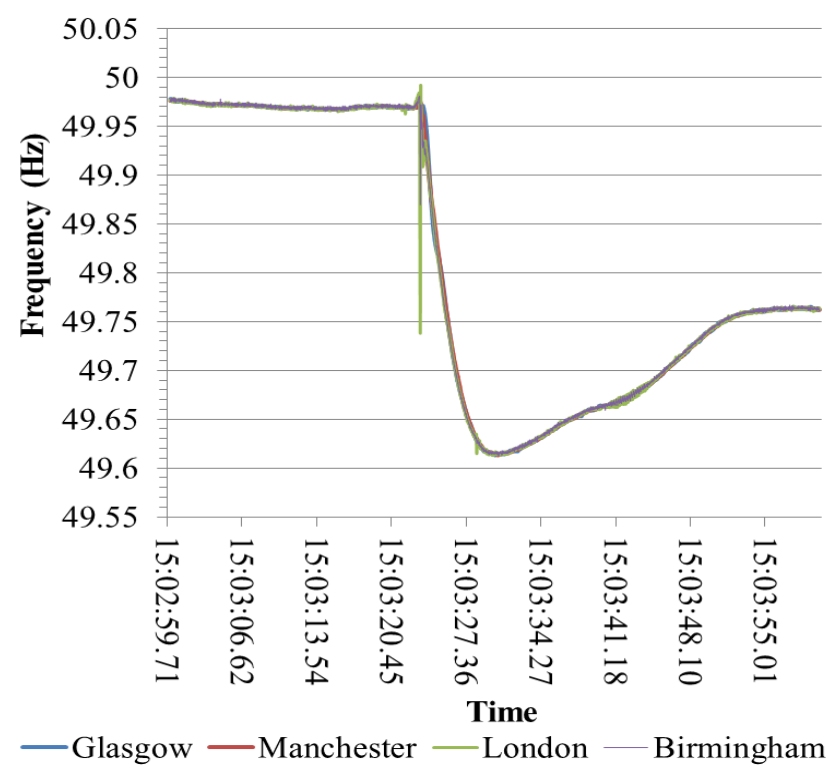

Fig. 4. UK frequency measured by four PMUs on 30/9/2012

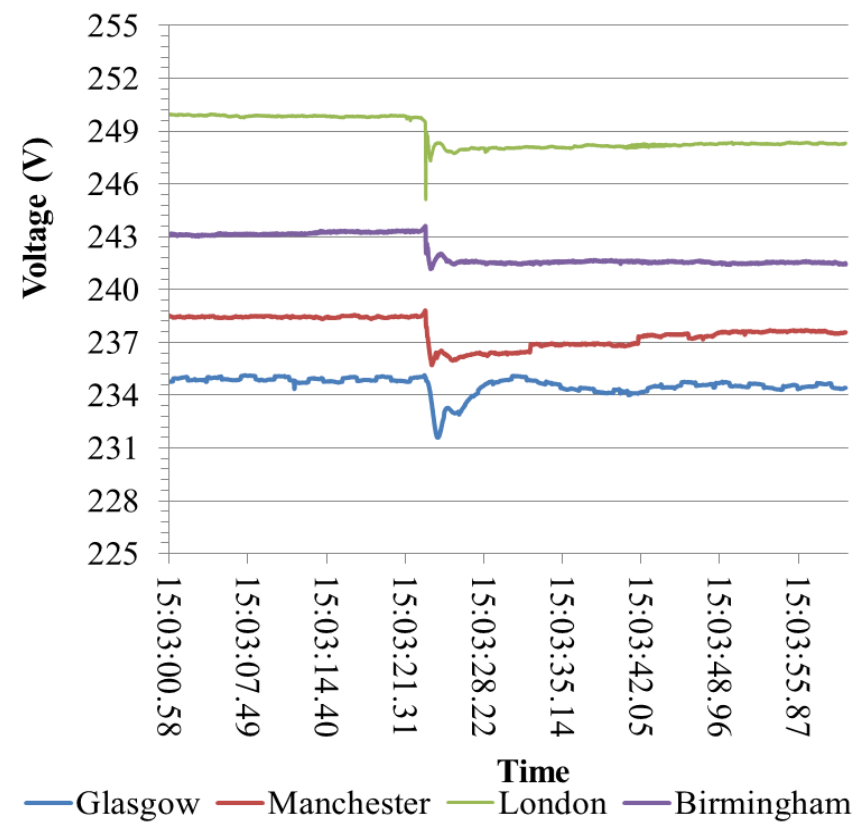

Fig. 5. Voltage magnitudes across the UK measured by four PMUs on $30 / 9 / 2012$

flows from the node with high phase angle toward the node with lower phase angle. The larger the phase angle difference between the two nodes, the greater the power flow between them. Exceeding of the power flow from a certain level can make the power system unstable. Therefore, a significant divergence in phase angle can be a sign of instability risk [26]. Figure 6 shows the phase angle deviation happened between Glasgow and London on 22 February 2013.

B. Schweitzer Engineering Laboratories (SEL) PDC

A local PDC has also been developed recently by using SEL-5073 PDC, which collects measurements locally [27]. 


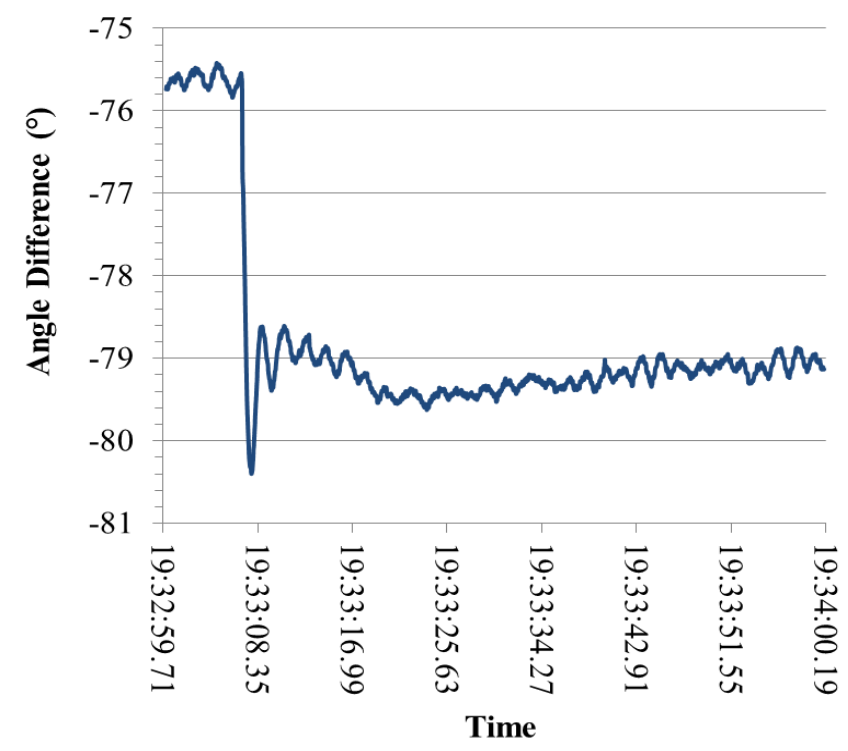

Fig. 6. Angle difference between Glasgow and London on 22/2/2013

SEL-5073 PDC software with integrated built-in archiving runs on windows-based computing platform and it is able to receive synchrophasor messages using Ethernet or serial communication. It can process incoming data from more than 500 PMUs at a maximum data rate of 240 messages per second. The processed data can be transmitted to up to six external clients as well as internal archive. The PDC Assistant software, a user-friendly interface, provides the ability to view the PDC real-time status, configure the PDC inputs, outputs, archives and so on.

SEL-5073 has high-speed calculation capability that can perform various phasor and analog calculations. This realtime measurement calculation function uses the measured parameters of the PMUs to calculate new parameters that are not directly collected, such as calculation of real and reactive power, derivatives, network latency etc. These calculations can be archived with other PMUs data or sent as an output stream.

SEL-5073 offers two types of archiving modes, triggered and continuous. The triggered archive stores data whenever an event happens, while continuous archive stores all received data. Another feature of the SEL-5073 is the archive retention setting, which enables deleting of archive data after specified duration. However, it offers Archive Collection Service (ACS) to back up the archive data and store data on a local or network storage drive automatically. For visualising and analysing real-time streaming and archive data SEL-5073 can be integrated with SEL-5078-2 Central software. The SEL Central allows a quick translate of synchrophasor data into visual information [27, 28].

\section{Open Source PDC}

An open source PDC has also been employed for further investigation. Earlier discussed PDCs were specialized standalone units and they were not openly available. But openPDC is an open source PDC, which was developed and made available to public by the Tennessee Valley Authority (TVA), in October 2009. OpenPDC architecturally consists of three layers: the Input, Action and Output Layers. Each layer performs a specific set of functions [29, 30].

The Input Adapter Layer reads streaming data from the measurement devices that may use different protocols. In fact, it allows the parsing of protocol and provides a generic data format. After assigning an ID to the measurement, it will transfer them to the Action Layer. Currently openPDC supports different kinds of protocols including IEEE C37.118-2005 and 2011, IEEE1344, BPA PDCStream, Virginia Tech FNET, Macrodyne, SEL Fast Message, etc. However, custom Input Adapters can be written to collect data from user desired input sources $[17,31]$.

The Action Adapter Layer deals with concentration and processing of the input measurements. One of the important functions of this layer is the phasor time alignment adapter. In this function the measurements received from the Input Layer are sorted by their associated GPS time-stamps and timealigned before transferring them to the next layer. The Action Adapter Layer also provides two more essential functions, which are real-time measurement calculation and real-time event detection.

Finally, the Output Adapter Layer of the openPDC receives all measured and calculated parameters at the end and queues up data and forwards them to either Historian system to archive them for future off-line analysis or to any other defined client systems. The Output Adapter can reencapsulate the data in several protocols. Example of openPDC output types are IEEE C37.118 concentrator output stream, Inter Control Center Protocol (ICCP), Comma Separated Values (CSV) File Export, and Historian Archiving output $[17,30]$.

OpenPDC has various features that make the monitoring of power systems more convenient. One of them is the ability to replay data stream from a specific time in the past. In this
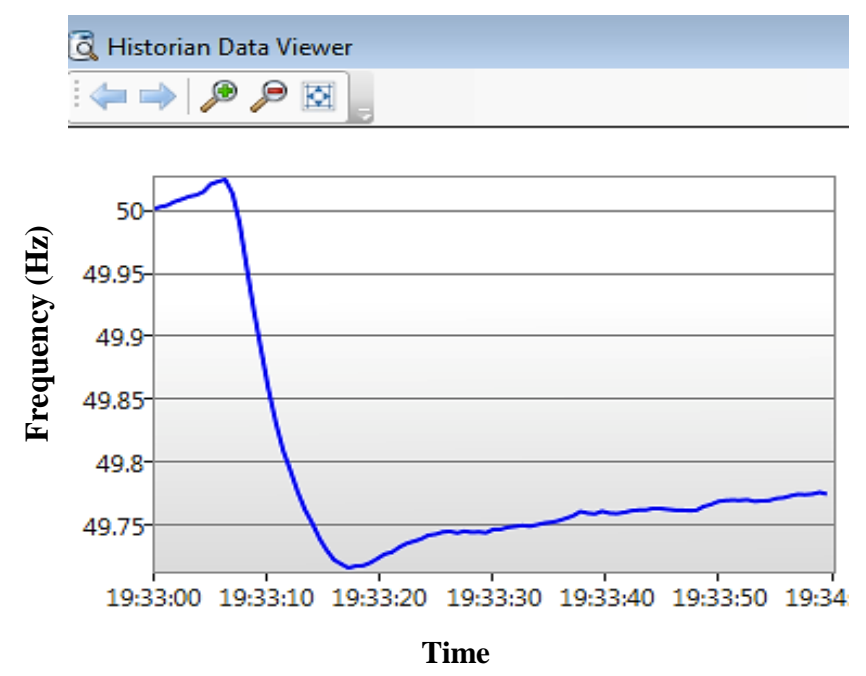

Fig. 7. London-Brunel University frequency captured by openPDC on $22 / 2 / 2013$ 
mode, the stored data from the Historian will be retransmitted to the openPDC software and shows the data as if it is coming from the PMU. The user can also speed up or slow down the replay speed. This near real-time event replay facilitates event analysis and helps the system operator to take appropriate corrective or protective control actions quickly. Another feature of openPDC is that each of the adapter layers functionalities can be improved or extended by user or developer. For example, a new model can be written for Input Adapter to enable support of new protocols. Or a new Action Adapter can be defined to provide particular function, detection of specific event, etc $[22,31]$. Figure 7 shows the frequency graph for Brunel University PMU, captured by openPDC on 22 February 2013.

\section{CONCLUSION}

WAMS capabilities make it an invaluable technology to enhance the reliability of power grids. In this paper, the main components of a WAMS alongside their requirements, technical challenges, and related technologies were discussed. A successful implementation of WAMS needs an elaborate plan and design of these components. Samples of events captured by laboratory-based employment of WAMS across the UK network were presented. Number of data concentrator software and their features were also investigated. Among them openPDC source codes are openly available, which enables users to extend and modify functionalities of the data concentrator.

\section{REFERENCES}

[1] R.B. Bobba, J. Dagle, E. Heine, H. Khurana, W.H. Sanders, P. Sauer, T. Yardley, "Enhancing Grid Measurements: Wide Area Measurement Systems, NASPInet, and Security," Power and Energy Magazine, IEEE , vol. 10, no. 1, pp. 67-73, Jan-Feb 2012.

[2] "Real-Time Application of Synchrophasors for Improving Reliability," NERC, 18 Oct., 2010, online: http://www.nerc.com.

[3] M. Gurbiel, P. Komarnicki, Z.A. Styczynski, M. Kereit, J. Blumschein, B.M. Buchholz, "Usage of Phasor Measurement Units for Industrial Applications," in Proc. Power and Energy Society General Meeting, July 2011, pp. 1-5.

[4] L. Vanfretti, M. Chenine, M.S. Almas, R. Leelaruji, L. Angquist, L. Nordstrom, "SmarTS Lab - A laboratory for developing applications for WAMPAC Systems," Power and Energy Society General Meeting, 2012 IEEE , pp.1-8, 22-26 July 2012.

[5] Elias Karam, "Implementation and Simulation of Communication Network for Wide Area Monitoring and control Systems in OPNET," Master of Science Thesis, KTH, Stockholm, Sweden, 2008.

[6] David G. Hart, David Uy, Vasudev Gharpure, Damir Novosel, Daniel Karlsson, Mehmet Kaba, "PMUs - A new approach to power network monitoring" ABB Article, 13 Dec. 2006, [Online] available at: http://www.abb.com/abblibrary/downloadcenter.

[7] J. De La Ree, V. Centeno, J.S. Thorp, A.G. Phadke, "Synchronized Phasor Measurement Applications in Power Systems," IEEE Transactions on Smart Grid, vol. 1, no. 1, pp. 20-27, June 2010.

[8] D.M. Laverty, R.J. Best, P. Brogan, I. Al Khatib, L. Vanfretti, D.J. Morrow, "The OpenPMU Platform for Open-Source Phasor Measurements," IEEE Transactions on Instrumentation and Measurement, vol. 62, no. 4, pp. 701-709, April 2013.

[9] "PMU Installation and Configuration Requirements," MISO, Version No. 1, 8 Sep. 2012, Online: https://www.misoenergy.org.
[10] "A Guide for PMU Installation, Commissioning and Maintenance Part II PMU Installation Procedures," NASPI, 5 June 2007, Online: https://www.naspi.org.

[11] Tianshu Bi, Hao Liu, Xing Zhou, Qixun Yang, "Impact of transient response of instrument transformers on phasor measurements," IEEE Power and Energy Society General Meeting, pp. 1-6, 25-29 July 2010.

[12] "Model 1133A Power Sentinel Operational Manual," Arbiter Systems, Online: http://www.arbiter.com.

[13] Mohammad Golshani, Gareth A. Taylor, Ioana Pisica, Phillip Ashton, "Investigation of open standards to enable interoperable wide area monitoring for transmission systems," Universities Power Engineering Conference (UPEC), 2012 47th International, pp.1-6, 4-7 Sept. 2012.

[14] A. Armenia, J.H. Chow, "A Flexible Phasor Data Concentrator Design Leveraging Existing Software Technologies," IEEE Transactions on Smart Grid, vol. 1, no. 1, pp. 73-81, June 2010.

[15] IEEE Std C37.118.2TM-2011, IEEE Standard for Synchrophasor Data Transfer for Power Systems, Dec. 2011

[16] Yee Wei Law, M. Palaniswami, G. Kounga, A. Lo, "WAKE: Key management scheme for wide-area measurement systems in smart grid," IEEE Communications Magazine, vol. 51, no. 1, pp. 34-41, Jan. 2013.

[17] M. Chenine, L. Vanfretti, S. Bengtsson, L. Nordstroum, "Implementation of an experimental wide-area monitoring platform for development of synchronized phasor measurement applications," IEEE Power and Energy Society General Meeting, pp.1-8, 24-29 July 2011.

[18] "Hadoop as the Platform for the Smartgrid at TVA," Cloudera, 26 Aug, 2010, online: http://www.cloudera.com/

[19] Mohammad Golshani, Gareth A. Taylor, Ioana Pisica, Phillip Ashton, "Laboratory-Based Deployment and Investigation of PMU and OpenPDC Capabilities" The $10^{\text {th }}$ International Conference on AC and DC Power Transmission (IET), December 2012, Birmingham, UK.

[20] A.G. Phadke, J.S. Thorp, "Communication needs for wide area measurement applications," Critical Infrastructure (CRIS), 5th International Conference, September 2010.

[21] V. Terzija, G. Valverde, Deyu Cai, P. Regulski, V. Madani, J. Fitch, S. Skok, M.M. Begovic, A. Phadke, "Wide-Area Monitoring, Protection, and Control of Future Electric Power Networks," Proceedings of the IEEE , vol. 99, no. 1, pp. 80-93, Jan. 2011.

[22] Guorui Zhang, Kai Sun, H. Chen, R. Carroll, Y. Liu, "Application of synchrophasor measurements for improving operator situational awareness, " IEEE Power and Energy Society General Meeting, pp.1-8, 24-29 July 2011.

[23] G. Zhang, S. Lee, R. Carroll, J. Zuo, L. Beard, Y. Liu, "Wide area power system visualization using real-time synchrophasor measurements," IEEE Power and Energy Society General Meeting, pp. 1-7, 25-29 July 2010

[24] Naduvathuparambil, B., Valenti, M.C., Feliachi, A., "Communication delays in wide area measurement systems," Proceedings of the ThirtyFourth Southeastern Symposium on System Theory, pp. 118-122, 2002.

[25] ELPROS, [http://www.elpros.si]

[26] M. Erol-Kantarci, H.T. Mouftah, "Smart grid forensic science: applications, challenges, and open issues," IEEE Communications Magazine, vol. 51, no. 1, pp. 68-74, January 2013.

[27] Schweitzer Engineering Laboratories (SEL), [https://www.selinc.com]

[28] "SEL-5073 SYNCHROWAVE Phasor Data Concentrator Instruction Manual," SEL, [https://www.selinc.com]

[29] L. Vanfretti and J.H. Chow, "Synchrophasor Data Applications for Wide-Area Systems," In Proceedings of the Power Systems Computation Conference 2011, Stockholm, Sweden.

[30] "OpenPDC: The Open Source Phasor Data Concentrator," online: http://openpdc.codeplex.com.

[31] P. Trachian, "Machine learning and windowed subsecond event detection on PMU data via Hadoop and the openPDC," IEEE Power and Energy Society General Meeting, pp. 1-5, 25-29 July 2010. 